\title{
Morphological patterns of the heteropycnotic chromatin and nucleolar material in meiosis and spermiogenesis of some Pentatomidae (Heteroptera)
}

\author{
Hederson Vinicius de Souza ${ }^{1}$, Márcia Maria Urbanin Castanhole ${ }^{1}$, \\ Hermione Elly Melara de Campos Bicudo ${ }^{1}$, Luiz Antônio Alves Costa $^{2}$ and Mary Massumi Itoyama ${ }^{1}$ \\ ${ }^{1}$ Laboratório de Citogenética de Insetos, Departamento de Biologia, Instituto de Biociências, \\ Letras e Ciências Exatas, Universidade Estadual Paulista, São José do Rio Preto, SP, Brazil. \\ ${ }^{2}$ Departamento de Entomologia, Museu Nacional, Universidade Federal do Rio de Janeiro, \\ Rio de Janeiro, RJ, Brazil.
}

\begin{abstract}
Pentatomidae is a family of Heteroptera which includes several agriculture pests that have had different aspects of their meiosis and spermiogenesis analyzed. In the present study we analyzed the morphological patterns of the heteropycnotic chromatin and the nucleolar material of Mormidea v-luteum, Oebalus poecilus and Oebalus ypsilongriseus. The three species presented multilobate testes, with three lobes in $M$. $v$-luteum and four in the Oebalus species. A karyotype with $2 n=14$ chromosomes $(12 A+X Y)$ was observed in the three species. Several characteristics were common to the three species, such as the absence of a testicular harlequin lobe (a lobe which produces different types of spermatozoa, previously considered a general characteristic of this family), late migration of the sex chromosomes and semi-persistence of the nucleolus. The three species also shared some characteristics regarding the patterns of the heteropycnotic chromatin and nucleolar material, but differed in others mainly related to the location of the heteropycnotic chromatin in the spermatids and the morphology and distribution of the nucleolar material at zygotene. The differences were always between species from different genera, suggesting a relationship with their genetic divergence.
\end{abstract}

Key words: nucleolus semi-persistence, late chromosome migration, multilobed testes.

Received: November 23, 2007; Accepted: March 13, 2008.

\section{Introduction}

Most Heteroptera feed on plantations or grains stored for human consumption during their nymph and adult stages, causing great economical losses. Heteroptera includes approximately 37,000 species distributed in eight infraorders, five of which contain noxious species (Rebagliati et al., 2005). The family Pentatomidae (infraorder Pentatomorpha), with eight subfamilies (Asopinae, Cyrtocorinae, Discocephalinae, Edessinae, Pentatominae, Phyllocephalinae, Podopinae and Serbaninae) and 4,112 species is among them (Schaefer and Panizzi, 2000). They include the "stink bugs" thus called due to their unpleasant smell produced by a gland that opens up in the metapleural region.

The presence of testes formed by a number of compartments referred to as "lobes" is a characteristic of the

Send correspondence to MM Itoyama. Laboratório de Citogenética de Insetos, Departamento de Biologia, Instituto de Biociências, Letras e Ciências Exatas, Universidade Estadual Paulista, Rua Cristóvão Colombo 2265, 15054-000 São José do Rio Preto, SP, Brazil. E-mail: mary@ibilce.unesp.br.
Pentatomidae. In some species, one of these lobes is of the harlequin type, which is differentiated from the other lobes by presenting spermatogonial cells with meiotic pairing, non-specific association of the autosomal bivalents, anomalous arrangement of the chromosomes in the metaphase plate, anomalous chromosome segregation and cell fusion, resulting in the production of spermatozoa with highly variable chromosome number (Rebagliati et al., 2005). There are reports of this type of lobe in 15 genera from three Pentatomidae subfamilies (Discocephalinae, Edessinae and Pentatominae) (Rebagliati et al., 2005).

In males of Pentatomidae the chromosome number varies from six to 27 , with 14 as the most frequent number $(85 \%)$. The sex chromosome system is $\mathrm{XX} / \mathrm{XY}$, except in three species: Macropygium reticulare $\left(\mathrm{X}_{1} \mathrm{X}_{2} \mathrm{Y}\right)$; Rhytidolomia senilis (Neo-XY) and Thyanta calceata $\left(\mathrm{X}_{1} \mathrm{X}_{2} \mathrm{Y}\right)$ (Rebagliatti et al., 2005). Pentatomidae karyotypes typically lack microchromosomes (Rebagliati et al., 2005, Lanzone and Souza, 2006; Souza et al., 2007a).

Pentatomidae basically share the chromosomal features present in other Heteroptera. These include holo- 
kinetic chromosomes, i.e., without a localized centromere, which causes microtubules to bind to the entire chromosome during mitosis leading to a parallel migration of the sister-chromatids to the cell poles at anaphase (Buck, 1968; Comings and Okada, 1972). At meiosis, however, the kinetic activity is restricted to the telomere regions and chromosomes are therefore called telokinetic (Motzko and Ruthmann, 1984). Meiotic behavior is different among autosomal bivalents and sex chromosomes (Ueshima, 1979; Manna, 1984; Papeschi and Mola, 1990; GonzálezGarcia et al., 1996; Suja et al., 2000). Except for a few species, autosomal bivalents are chiasmatic and segregate pre-reductionally (Nokkala and Grozeva, 2000). On the other hand, the sex chromosomes are achiasmatic and behave as univalents at male meiosis, equationally dividing at anaphase I and associating to form a pseudobivalent at meiosis II (nevertheless, the pre-reduction of sex chromosomes is also reported in some Pentatomidae species) (Ueshima, 1979; Grozeva and Nokkala, 2001).

More detailed cytogenetic aspects of Pentatomidae, such as morphological variations of the condensed chromatin and nucleolar material during cell division, have received little attention. Aiming at contributing to the cytogenetic characterization of Pentatomidae of economic interest, we analyzed some aspects of meiosis and spermiogenesis of Mormidea v-luteum, Oebalus poecilus and O. ypsilongriseus.

\section{Material and Methods}

Fifteen adult males of each of the species Mormidea v-luteum, Oebalus poecilus and Oebalus ypsilongriseus (Heteroptera, Pentatomidae, Pentatominae, Pentatomini) were colected from okra trees in São José do Rio Preto (2047'32" S, 49²1'37" W), São Paulo State, Brazil. The specimens were fixed in methanol:acetic acid (3:1), their testicular lobes were isolated and squashed before staining with lacto-acetic orcein. The pattern of the heteropycnotic chromatin in testicular cells was then analyzed. The nucleolar morphology was analyzed after impregnation with silver nitrate (Howell and Black, 1980). Both kinds of analyses were performed during meiosis and spermiogenesis. Images were obtained under a Zeiss microscope with the AXIO VISION software.

\section{Results}

The three species presented multilobed testes covered by a reddish membrane. While three lobes were present in Mormidea v-luteum, four lobes were observed in the two Oebalus species. None of these species showed lobes with morphological characteristics of the harlequin type. In all of them, the mechanism of sex determination in males was $\mathrm{XY}$ and the karyotype was $2 \mathrm{n}=14(12 \mathrm{~A}+\mathrm{XY})$.

\section{Morphological variation of the heteropycnotic chromatin}

The term heterochromatin includes some characteristics which were not analyzed in this study and we thus use 'heteropycnotic' or 'condensed chromatin' to refer to the regions that are more intensely stained by lacto-acetic orcein.

In the three analyzed species, the polyploid nuclei of the nutritive cells were large, with a larger heteropycnotic region and several much smaller ones (Figure 1A). The whole process of spermatogenesis occurred in the spermatogonial cysts constituted by nine spermatocytes, each with several heteropycnotic regions (Figure 1B). In the three species, the cells at the initial prophase I (leptotene, zygotene and pachytene) exhibited a strongly stained heteropycnotic region, which was closer to the nucleus periphery and possibly composed of the $\mathrm{X}$ and $\mathrm{Y}$ chromosomes (Figure 1C-E). During diplotene and diakinesis, chiasmata were observed in the autosomal bivalents, but not in the sex chromosomes (Figure 1F-G). The X chromosome was larger than the Y (Figure 1H) and the sex chromosomes were seen together or separated at metaphase I (Figure 1H,I). In a polar view during metaphase I and II, the autosomes were arranged in a ring surrounding the sex chromosomes (Figure 1I). Late migration of the chromosomes was observed at anaphase and telophase I and II (Figure 1J-L).

Observations on the spermiogenesis of the three species showed that, in the initial round-shaped spermatids, the heteropycnotic chromatin is C-shaped and located in the internal part of the nuclear envelope, covering over half of it. A heteropycnotic corpuscle was also observed inside the spermatid nucleus of M. v-luteum, but not in the Oebalus species (Figure 1M, N). During spermatid elongation, the condensed chromatin remained close to the nuclear envelope in M. v-luteum (Figure 1O), while it became more central in $O$. poecilus and O. ypsilongriseus, resembling a longitudinal line (Figure 1P).

\section{Morphological variation of the nucleolar material}

A large irregular nucleolar mass, apparently formed by the association of smaller bodies, and some smaller corpuscles, were present in the polyploid nuclei of the nutritive cells of the three species (Figure 2A). A single nucleolar corpuscle was observed at the nucleus periphery of spermatocytes at initial prophase I (leptotene) in the three species (Figure 2B). The nucleolar corpuscle at zygotene in Oebalus increased in size and acquired an unusual mushroom-like form, with a larger, intensely stained part (the "hat") and a smaller fainter portion (the "stem") (Figure 2C). This morphological type of nucleolar material persisted during pachytene, when another round, smaller and intensely stained nucleolar body also appeared (Figure 2D). An apparent disintegration of the nucleolar material started between pachytene and diplotene, when round corpuscles 
of different sizes were seen (Figure 2E). Mormidea $v$-luteum differed from the other two species by the absence of this mushroom-like nucleolar structure. Instead, we only observed a nucleolar corpuscle and fibrilar structures associated with the chromosomes that later became dispersed in a granular form in this species (Figure $2 \mathrm{~F}, \mathrm{G}$ ). In the three

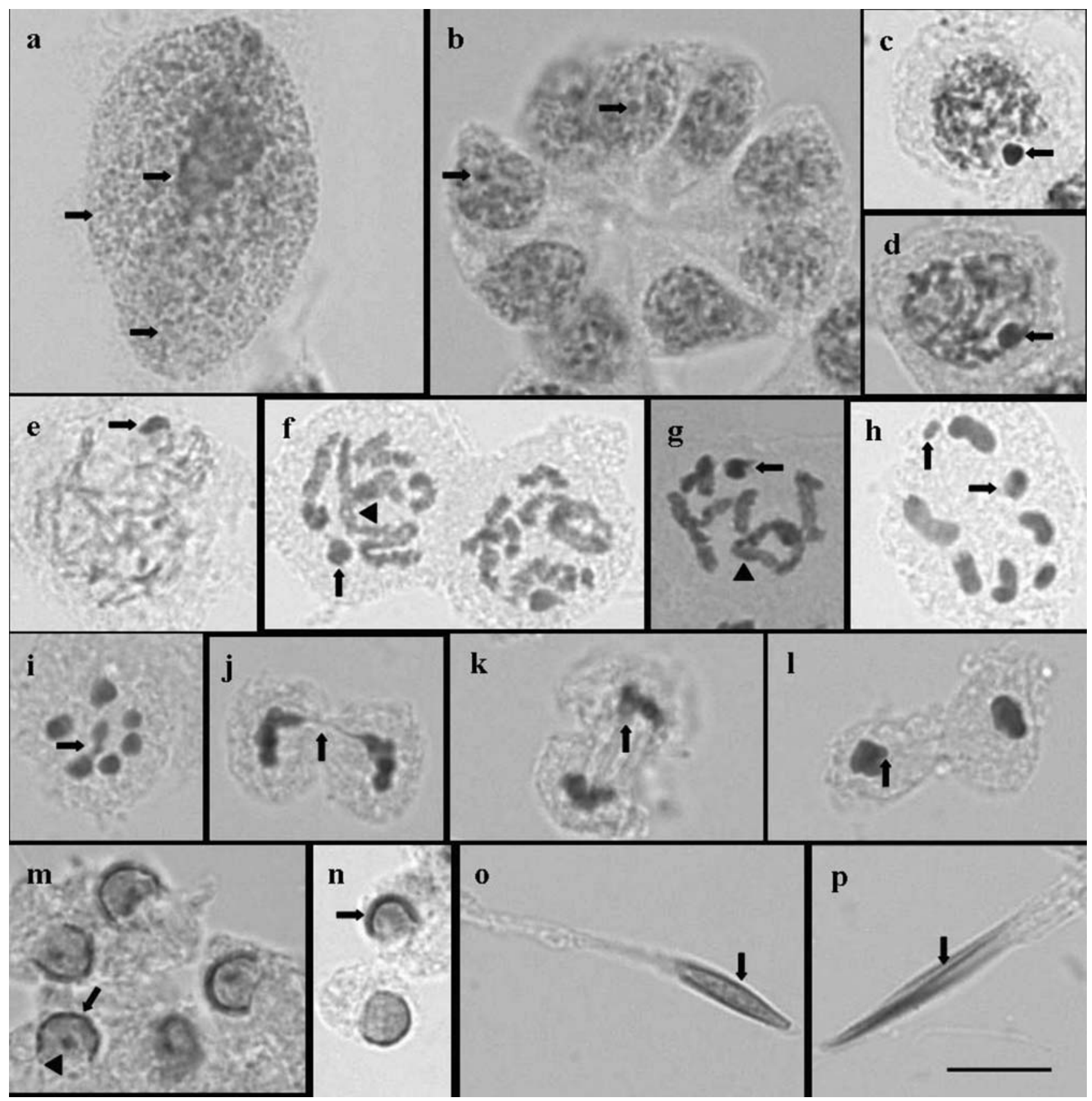

Figure 1 - Seminiferous tubule cells of Mormidea v-luteum (D, K, M and O), Oebalus poecilus (A, F, G, H, I, J, L, N and P) and Oebalus ypsilongriseus (B, C and E) adult males stained with lacto-acetic orcein. A) Polyploid nuclei of nutritive cells containing one large and many small heteropycnotic corpuscles (arrows); B) Spermatogonial cyst constituted by nine spermatocytes, each one containing a heteropycnotic body (arrows); C-G) The single heteropycnotic corpuscle (arrow) persists during the entire prophase I (in the sequence: leptotene, zygotene, pachytene, diplotene and diakinesis); note the presence of chiasmata in F and G (arrowheads); H) Metaphase I showing $2 \mathrm{n}=12 \mathrm{~A}+\mathrm{XY}$, the X (large) and $\mathrm{Y}$ (small) chromosomes are indicated by arrows; I) Polar view of metaphase I, the autosomal bivalents forming a ring around the sex chromosomes (arrow); J-L) Anaphases and telophase I; note the late migration of the chromosomes (arrows); M) In round spermatids of Mormidea v-luteum, the heteropycnotic chromatin is present in the center of the nucleus forming a single corpuscle (arrowhead) and around the interior of the nucleus envelope arranged in a $\mathrm{C}$ - shaped structure (arrow); $\mathbf{N}$ ) In the round spermatid of Oebalus species the heteropycnotic chromatin is exclusively present in the interior of the nucleus (arrow); O) Elongated spermatid of Mormidea showing the heteropycnotic chromatin maintained at the nucleus periphery (arrow); P) Elongated spermatid of Oebalus showing the heteropycnotic chromatin as a longitudinal line in the interior of the head (arrow). Bar $=10 \mu \mathrm{m}$. 
species, the nucleolar material could be observed as a round corpuscle until the end of telophase I (Figure 2H-K) and the still round spermatids exhibited a variable number of small nucleoli, some of which were more intensely stained (Fig- ure 2L-N). When the spermatids started to elongate, the silver nitrate-stained material concentrated at the posterior region of the head, where it was seen until the formation of the spermatozoa (Figure 2O-S).

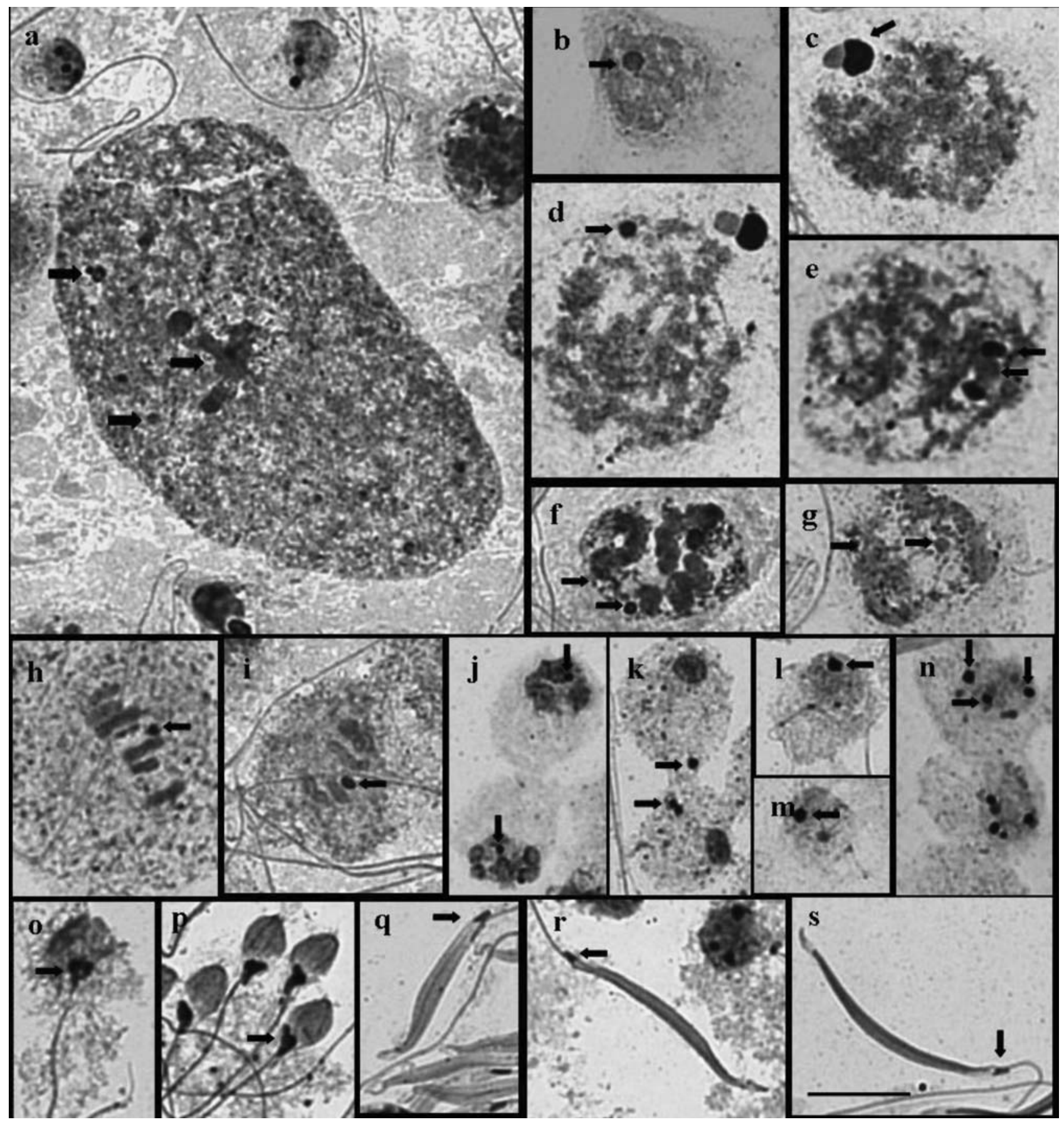

Figure 2 - Seminiferous tubule cells of Mormidea v-luteum (A, B, F, G, I and N), Oebalus poecilus (L, M, P and R) and Oebalus ypsilongriseus (C, D, E, $\mathrm{H}, \mathrm{J}, \mathrm{K}, \mathrm{O}, \mathrm{Q}$ and S) adult males, impregnated by silver nitrate. A) Polyploid nuclei of nutritive cells containing a large mass of nucleolar material and several small corpuscles (arrows); B) One large nucleolar corpuscle is present in the cell nuclei at leptotene (arrow); C, D) In both Oebalus species two associated nucleolar bodies are present at zygotene, one more impregnated than the other and showing a mushroom-like shape (C, arrow); note a small additional corpuscle in D (arrow); E-G) Beginning of the disorganization process of the nucleolar material at pachytene/diplotene (arrows); $\mathbf{H}, \mathbf{I})$ metaphases I in lateral view with the nucleolar material still present (arrows); J, K) nucleolar material also present at telophase (arrows); L-N) Round spermatids with a variable number of nucleolar bodies (arrows); O-S) Spermatids with different degrees of elongation; the nucleolar material is located in the posterior region of the head (arrows). Bar $=10 \mu \mathrm{m}$. 


\section{Discussion}

We studied the morphological changes of the heteropycnotic chromatin and of the nucleolar material during meiosis and spermiogenesis of Mormidea v-luteum, Oebalus poecilus and O. ypsilongriseus. Changes in the amount and distribution of decondensed and condensed chromatin may reflect genetically active and inactive chromosome regions, respectively, while morphological alterations of the nucleolus (including number and structure) reflect the pattern of ribosome production, a central process in the protein synthesis.

We observed similarities and differences among the species studied. The three species had multilobed testes with three lobes in M. v-luteum and four in O. poecilus and O. ypsilongriseus. Multilobed testes are a characteristic of the Pentatomidae, but the number of lobes observed in the species analyzed herein was smaller than that typically found in the family, which is seven. In addition, the three species differed from the other 27 species of the same family and subfamily (Pentatominae) already studied by the absence of a harlequin lobe (Rebagliati et al., 2005; Lanzone and Souza, 2006; Souza et al., 2007a). The Pentatomidae Antiteuchus tripterus also showed less lobes (six) than the typical seven, but one of them was harlequin (Souza et al., 2007a).

Rebagliati et al. (2005) considered the development of harlequin lobes as dependent on the environment in which the insects live. However, the harlequin lobe was observed in samples of Antiteuchus tripterus collected in apparently different environments in several states in Brazil (São Paulo, Souza et al., 2007a; Pernambuco, Alagoas and Bahia, Lanzone and Souza, 2006). These observations suggest that the presence of that lobe is a genetic characteristic of the species rather than an environmental effect.

Another feature shared by the three species was the $2 n=14$ karyotype, with $\mathrm{XY}$ males, suggesting a $\mathrm{XX} / \mathrm{XY}$ sex chromosome system, found in most previously analyzed Pentatomidae.

The distribution of the heteropycnotic chromatin during meiosis and spermiogenesis was common to the three species, except in the round spermatids, in which $M$. $v$-luteum exhibited an additional corpuscle inside the head, not observed in the other species. The distribution of the heteropycnotic chromatin in elongated spermatids in $M$. $v$-luteum also differed from that of the other species, being close to the membrane in the interior of the head, while it formed a filament in the central region in Oebalus.

As to the nucleolar activity, in some insects (including some Heteroptera) the silver nitrate specifically stains the previously active nucleolar organizing regions (NORs), which are chromosome sites of rDNA. In some Heteroptera, a single NOR was observed, which could be interstitial or terminal and located at autosomes or sex chromosomes (Camacho et al., 1985; Fossey and Liebenberg, 1995; Gon-
zález-García et al., 1996; Papeschi et al., 2003; Rebagliati et al., 2003).

In the three Pentatomidae species studied herein no NORs could be evidenced, even after some technical variations were tried. Nevertheless, after silver staining, the morphological changes of the nucleolar material could be analyzed. The three species apparently did not differ as to the pattern observed in the nutritive cells. The nucleolar material remained visible in the three species during the entire meiosis and spermiogenesis, a phenomenon called nucleolar semi-persistence (Pickett-Heaps, 1970).

Nucleolar semi-persistence differs from the more general process in which the disorganization of the nucleolus occurs during prophase, so that from diakinesis until the end of the meiotic division, this organoid is not visible, suggesting that, at that time, it is already completely disorganized (Risueño and Medina, 1976). Among Heteroptera, exceptions to this general behavior were described for other species, such as Nysius californicus (Lygaeidae, Souza et al., 2007b) and Antiteuchus tripterus (Pentatomidae, Souza et al., 2007a). In both cases, silver-stained material was observed around the chromosomes until the meiotic metaphase I.

Differences in the nucleolar material among the three species were detected at zygotene and pachytene, with the Oebalus species showing a "mushroom-like" structure. In the same meiotic stages, M. v-luteum presented a single nucleolar corpuscle with size and staining similar to the fainter part of the "mushroom-like" structure of Oebalus.

The nucleolar semi-persistence is another structural characteristic of the meiosis which, similarly to the presence of the harlequim lobe, has been attributed to environmental effects. According to Cattani and Papeschi (2004), the nucleolar semi-persistence was detected in individuals of the Heteroptera Spartocera fusca that spent more time in the field, being thus longer exposed to environmental factors such as fluctuations of temperature, humidity and precipitation. We do not have such information on the species that we studied, but they were collected in the same place at the same time.

In the present study, the differences observed were between species belonging to different genera. They may thus be part of the genetic features that evolved during the divergence of the two genera, reflecting physiological differences that remain to be understood.

\section{Acknowledgments}

Thanks are due to Dr. Sonia Maria Oliani (Department of Biology - IBILCE/UNESP) for the opportunity of capturing cell images and to FAPESP, FUNDUNESP and CNPq for financial support. 


\section{References}

Buck RC (1968) Mitosis and meiosis in Rhodnius prolixus: The fine structure of the spindle and diffuse kinetochore. J Ultrastruct Res 18:489-501.

Camacho JPM, Belda J and Cabrero J (1985) Meiotic behaviour of the holocentric chromosomes of Nezara viridula (Insecta, Heteroptera) analyzed C-banding and silver impregnation. Can J Genet Cytol 27:491-497.

Cattani MV and Papeschi AG (2004) Nucleolus organizing regions and semi-persistent nucleolus during meiosis in Spartocera fusca (Thunberg) (Coreidae, Heteroptera). Hereditas 140:105-111.

Comings DE and Okada TA (1972) Holocentric chromosomes in Oncopeltus: kinetochore plates are present in mitosis but absent in meiosis. Chromosoma 37:177-192.

Fossey A and Liebenberg H (1995) Meiosis and nucleolar structures in the stink bug Carlisis wahlbergi Stal (Coreidae, Heteroptera). Cytobios 81:7-15.

González-Garcia JM, Antonio C, Suja JÁ and Rufas JS (1996) Meiosis in holocentric chromosomes: Kinetic activity is randomly restricted to the chromatid ends of sex univalents in Graphosoma italicum (Heteroptera). Chromosome Res 4:124-132.

Grozeva S and Nokkala S (2001) Chromosome numbers, sex determining systems, and patterns of the C-heterochromatin distribution in 13 species de Lace Bugs (Heteroptera, Tingidae). Folia Biol 49:29-41.

Howell WM and Black DA (1980) Controlled silver staining of nucleolus organizer regions with protective colloidal developer: 1-step method. Experientia 36:1014-1015.

Lanzone C and Souza MJ (2006) Chromosome complement and meiosis in the three species of the Neotropical bug genus Antiteuchus (Heteroptera, Pentatomidae, Discocephalinae). Genet Mol Biol 29:49-55.

Manna GK (1984) Chromosomes in evolution in Heteroptera. In: Sharma AK (ed) Chromosomes in Evolution of Eukaryotic Groups. CRC Press, Boca Raton, pp 189-225.

Motzko D and Ruthmann A (1984) Spindle membranes in mitosis and meiosis of the heteropteran insect Dysdercus intermedius. A study of the interrelationship of spindle architecture and the kinetic organization of chromosomes. Eur J Cell Biol 33:205-216.
Nokkala S and Grozeva S (2000) Achiasmatic male meioses in Myrmedobia coleoptratal (Fn.) (Heteroptera, Microphysidae). Caryologia 53:5-8.

Papeschi AG and Mola LM (1990) Meiotic studies in Acanonicus hahni (Coreidae, Heteroptera). I. Behavior of univalents in desynaptic individuals. Genetica 80:31-38.

Papeschi AG, Mola LM, Bressa MJ, Greizerstein EJ, Lía V and Poggio L (2003) Behaviour of ring bivalents in holocentric systems: Alternative sites of spindle attachment in Pachylis argentinus and Nezara viridula (Heteroptera). Chromosome Res 11:725-733.

Pickett-Heaps JD (1970) The behavior of the nucleolus during mitosis in plants. Cytobios 6:69-78.

Rebagliati P, Papeschi AG and Mola LM (2003) Meiosis and fluorescent banding in Edessa meditabunda and $E$. rufomarginata (Heteroptera, Pentatomidae, Edessinae). Eur J Entomol 100:11-18.

Rebagliati PJ, Mola LM, Papeschi AG and Grazia J (2005) Cytogenetic studies in Pentatomidae (Heteroptera): A review. J Zool Syst Evol Res 43:199-213.

Risueño MC and Medina FJ (1976) The nucleolar structure in plant cells. Rev Biol Cel 7:1-162.

Schaefer WC and Panizzi AR (2000) Heteroptera of economic importance. Boca Raton, CRC Press, 824 pp.

Souza HV, Bicudo HEMC and Itoyama MM (2007b) Study of chromosomal and nucleolar aspects in testes of Nysius californicus (Heteroptera, Lygaeidae). Genet Mol Res 6:33-40.

Souza HV, Bicudo HEMC, Costa LAA and Itoyama MM (2007a) A study of meiosis and spermatogenesis in different testicular lobes of Antiteuchus tripterus (Heteroptera, Pentatomidae). Eur J Entomol 104:353-362.

Suja JA, Del Cerro AL, Page J, Rufas JS and Santos JL (2000) Meiotic sister chromatid cohesion in holocentric sex chromosomes of three heteropteran species is maintained in absence of axial elements. Chromosoma 109:35-43.

Ueshima N (1979) Animal Cytogenetics. v. 3. Insecta 6. Hemiptera, Heteroptera. Gebr. Borntraeger, Berlin, $119 \mathrm{pp}$.

Associate Editor: Yatiyo Yonenaga-Yassuda

License information: This is an open-access article distributed under the terms of the Creative Commons Attribution License, which permits unrestricted use, distribution, and reproduction in any medium, provided the original work is properly cited. 